\title{
Surface Modification of Cotton Fiber
}

\author{
Derseh Yilie limeneh ${ }^{1 *}$ and Kelem Tiessasie Yilma ${ }^{2}$ \\ ${ }^{1}$ Textile Manufacturing, Ethiopian Institute of Textile and Fashion Technology, Bahir Dar University, Ethiopia \\ ${ }^{2}$ Fiber Science and Technology, Ethiopian Institute of Textile and Fashion Technology, Bahir Dar University, Ethiopia
}

*Corresponding author: Derseh Yilie limeneh, Textile Manufacturing, Ethiopian Institute of Textile and Fashion Technology, Bahir Dar University, Ethiopia.

Received Date: May 23, 2020

Published Date: June 11, 2020

\begin{abstract}
Cotton weather in the form of fiber or fabric, a natural cellulose material, is widely used in the textile industry for its excellent properties. However, its application in some fields is seriously restricted because of its poor ant pilling behavior, antibacterial and UV-protection, comfortably, tensile properties, softness properties, water repellence and wrinkle recovery, hydrophobic property, wicking properties and dye ability of cotton fabrics and to use it as nano particle. That is why the surface and chemical modification carried out through different methodology with chemicals used were of analytical reagent grade to achieve such important property of the cotton materials like super hydrophobic, good surface property of a cotton when it treat with weather plasma, nanoparticles coating, composite film and chemical treatments (3-glycidoxypropyltriethoxysilane,bifunctional polysiloxanes, silane coupling agents vinyltriethoxysilane and aminopropyltriethoxysilane, chloropyrimidine compounds. All modification enhances the application of cotton material in different sectors.
\end{abstract}

Keywords: Hydrophobic property; Surface modification; Chemical modification; Wicking property; Functional property

\section{Introduction}

Cotton fiber is one of the most important natural fibers which provide a wide range of application in textile materials because of its easy availability, low density, light weight, low cost, and above all environment friendly characteristics. But the major problem of the cotton fiber is its lower flexibility and softness properties which limit an extended use of cotton as well as other fibers. By these modifications, some new moieties are introduced on the fiber backbone that can improve its properties. That is why the surface modification of cotton fiber was successfully carried out by condensation polymerization with 3-glycidoxypropyltriethoxysilane (GPTES) in an ethanol-water medium to enhance the tensile strength and softness properties of the cotton, by introducing a more flexible $\mathrm{Si}-\mathrm{O}$ bond between the silane coupling agents and the cotton fiber was introduced by Mondal IH, et al. [1]. Other report in 2014 by Bhat, et al. [2] declare that effect of plasma treatments on the surface properties of textile fibers by using the radio frequency (RF)-generated plasma in terms of chemical interactions on the surface modification of cotton fabrics using a variety of gases and its effects on wetting and dyeing properties. Like other modification hydrophobicity of cotton can be done by Zhang M, et al. [3] in which the modification can effectively protect the cotton fabric from pollution, mildew and shrinkage which is greatly restrict the further application and development of cotton textile by treating cotton surface with zinc oxide film. Since highly hydrophobic natural textiles due their unique characteristics such as self-cleaning, anti-contamination and anti-sticking needed so fabrication of super hydrophobic cotton fabrics by a simple chemical modification using bifunctional polysiloxanes with various contents of functional groups can be demonstrated by Przybylak M. et al. [4]. Chemical treatment of the cotton fibers not only reduces their moisture absorption process but also functionalize a cotton fiber. Mondal IH, etal. [5] investigates the effect of silane treatment on the moisture resistance, swelling behavior, tensile strength, wrinkle 
recovery properties, thermal stability and surface morphology of cotton fibers. Much less has been reported on modification of cotton fiber by silane coupling agents. In investigation by Kongdee A, et al. [6] modification of Cotton Fibers with Sericin Using NonFormaldehyde Released Cross linking Agents are done in which the cotton fibers were selected to keep moisture absorbency; they were modified with sericin for biomedical purposes.

Composite material with natural cellulosic fibers had attached a lot of attention due to their biodegradability, excellent mechanical property and high surface reactivity. But the one which is declared by Patino-ruiz D, et al. [7] can improve its application by modify cotton fibers with magnetite and magnetic core-shell mesoporous silica nanoparticles. Functional finishing of cotton fabrics using zinc oxide-soluble starch nanocomposites to impart antibacterial and UV-protection functions also be reported by Vigneshwaran N, et al. [8], which is better way to solve such a problem with cotton fabric. Cotton fiber easily unravel and the loose fiber ends form balls on the surface of fabric during the process of dyeing and finishing, wearing, and washing, which effects the handle, appearance, and wear ability of fabrics. For this Dong X, et al. [9] finds that the poor ant pilling behavior of cotton fabric with durable antipilling modification of cotton fabric with chloropyrimidine compounds. The effect of gamma radiation on the cotton fabric and compare the dye ability of gamma irradiated fabric with that of chemically mercerized fabric using reactive dye, reactive violet H3R is investigated by Zahid M, et al. [10] and the color strength values for the mercerized and the gamma irradiated cotton fabric show that the irradiated fabric had high color strength at $60^{\circ} \mathrm{C}$ using dye bath of pH10 in the presence of $6 \mathrm{~g} / \mathrm{L}$ of exhausting agent while dyeing for $40 \mathrm{~min}$. according to this work both mercerization and irradiation increased the surface area of fibers that substantially elevated the dyeing performance and fastness properties. The objective of this work is to investigate the functional modification of cotton fiber or fabrics.

\section{Materials and Methods}

\section{For textile performance of functionalized cotton fiber with 3-glycidoxypropyltriethoxysilane}

Cotton fiber sample with3-Glycidoxypropyltriethoxysilane chemical were used to for the evaluation of textile performance of functionalized cotton fiber with 3-Glycidoxypropyltriethoxysilane by Mondal IH, et al. [1]. Alkaline washing was applied for the removal of non-cellulose compounds before cotton fibers were dipped in silane solution, prepared by mixing GPTES with an ethanol/water mixture, in which evaluation of physical and chemical Properties such as Measurement of tensile strength (portable Electronic Single Yarn Strength Tester YG021J), moisture analyzers(moisture content), swelling behavior(dipping them in water, methanol, and carbon tetrachloride), wrinkle recovery angle (wrinkle recovery tester) of the functionalized and unmodified cotton fibers. Finally characterization of Unmodified and Surface Modified Cotton Fibers are done by using infrared spectroscopy, scanning electron microscopy analysis, thermo-gravimetric analysis, XRD analysis to determine the composition, microstructure and the surface morphology, thermal decomposition rate and the thermal stability, diffraction intensity respectively.

\section{For surface modification of cotton fabrics using plasma technology}

The gray cotton fabric with different type of dyes (direct reactive and neutral) used were for the surface modification of cotton fabrics using plasma treatment with Bhat NV, et al. [2]. A sample of size $20 \mathrm{~cm}$ times $20 \mathrm{~cm}$ was inserted in the chamber and treated by plasma. The main gases, pressure were air and dichlorodifluoromethane (DCFM) and 20 Pascal was maintained during the plasma treatments. Gray, as well as desized, scoured, and bleached cotton, fabrics were used for further studies and subjected to plasma treatments the surface morphologies of treated fabrics were examined using a scanning electron microscope (model JEOL, JSM-5400), absorbency (AATCC 79-2000), contact angles (photographs of the water drops), wicking action (ISO 90736:2000) and after dyeing of desized, scoured, and plasma-treated fabrics then the amount of dye absorbed by the sample, color strength using an ultraviolet/visible spectrophotometer and Nova Scan, Macbeth Color Eye 7000A equipment respectively.

\section{For super hydrophobic cotton textile with robust composite film and flame retardancy}

Zhang M, et al. [3], prepare zinc oxide film super hydrophobic cotton textile with robust composite film and flame retardancy by using cotton sample immersed in amine zinc oxide solution (zinc oxide added to ethanol in the presence of APDMS), would solidly stick to the cotton substrate via a chemical bonding of numerous amino and epoxy groups through then the dried was fluorinated using trimethethoxysilane solution. Ultimately the super hydrophobic cotton fabrics were obtained at ambient temperature and to enhance the mechanical stability, polystyrene was introduced to further decorate the super-hydrophobic cotton surface. Then the morphology of the cotton sample surface and zinc oxide was characterized with scanning electron microscope (FEI QUANTA200), transmission electrons microscope (JEOL JEM2100). The pure and amine-functionalized zinc oxide, elemental composition of the film on the sample surface, water contact angle, abrasion, thermo gravimetric analysis was analyzed by Fourier transform infrared spectroscopy (MAGNA-IR560, Nicolet), X-ray photoelectrons spectroscopy (K-Alpha), contact meter(Powereach,JC2000C), Martindale ,thermo gravimetric analyzer CTA instrument) to measure abrasions as well as thermogravimetric used.

\section{For fabrication of super hydrophobic cotton fabrics by a simple chemical modification}

A cotton fabric with and two types of bifunctional polysiloxanes with different ratios of functional groups were used by Przybylak 
M. et al. [4] to fabrication of super hydrophobic cotton fabrics by a simple chemical modification and was performed by the onestep method via chemical treatment with solutions of bifunctional polysiloxanes or by the two-step method, which consisted of the introduction of silica sol at the first stage followed by the chemical modification. The durability of the hydrophobic properties of fabrics was determined by measurements of the WCA on the surface of fabrics after the modification and after one and ten washing. The analytical balance Ohaus, automatic video contact-angle testing apparatus ( Kruss model DSA 100), was used for determination of the amount of modifiers add-on and the analysis was carried out by employing the SEM-EDS technique to determine ultimate elements (Si,F, N and P) and water contact angles. Surface topography was carried out using a Hitachi S-3400 N scanning electron microscope.

\section{For modification of cotton fiber with functionalized silane coupling agents vinyltriethoxysilane and aminopropyltriethoxysilane}

Silane treatment of cotton fibers was carried out with vinyltriethoxysilane and aminopropyltriethoxysilane after cotton fibers were washed with $0.2 \% \mathrm{Na} 2 \mathrm{CO} 3$ solution at $75{ }^{\circ} \mathrm{C}$ for 30 minutes then evaluation of physical properties such as tensile strength, moisture absorption, swelling capacity, wrinkle recovery angle, dyeing of raw and exhaustion of dye of silane modified cotton fibers was measured using a "Portable Electronic Single Yarn Strength Tester YG021J" and as a function of weight gain, treating them with water, methanol, and carbon tetrachloride, wrinkle recovery tester (Daiei Kagaku Seiki Ltd. Kyoto, JAPAN), dyeing machine (DYSIN, Taiwan, China), calorimetrically (Type-S104, No221 , Spectrophotometer) then finally characterization of surface modified cotton fibers is conducted by Infrared spectroscopy, scanning electron microscopy analysis, Thermogravimetricanalysis, Energy dispersive X-ray analysis using Perkin Elmer Spectrum 100 infrared spectrometer, electron microscope (FEI Quanta Inspect, Model: S50, Kyoto, Japan) to observe the microstructure and the surface morphology, Seiko-Extar-TG/DTA-6300 (Seiko-Japan), solid state device (FEI Quanta Inspect, Model: S50).

\section{For modification of cotton fibers with sericinusing non- formaldehyde released cross linking agents}

Different chemicals and cotton fabric sample $(30 \times 40 \mathrm{~cm} 2)$ was treated with finishing solutions, composed of glutaraldehyde, DMeDHEU and sericin and fabrics were padded through the finishing solutions without sericin added, and it was treated as control were done by Kongdee A, et al. [6] on modification of Cotton Fibers with Sericin Using Non-Formaldehyde Released Cross linking Agents then characterize and analyses treated cotton fabrics with A Vector 22 FTIR spectrophotometer, scanning electron microscopy (Jeol, JSM5410LV, Japan) as well as Data Color 650 spectrophotometer (USA) and after air drying, the color strength, and L- and b-values, of samples were measured using Data Color 650 spectrophotometer (USA) for measurement of Dyeing samples with acid dye.

\section{For modification of cotton fibers with magnetite and magnetic core-shell mesoporous silica nanoparticles}

David patino-ruiz et al., 2018, modification of cotton fibers with magnetite and magnetic core-shell mesoporous silica nanoparticles were declared by using different chemicals to synthesize magnetic nanoparticles according to the copricipitation methods and the magnetic core-shall mesoporous silica nanoparticles were synthesized adapted from previous report then the cotton fiber were cleaned and modified prior to nanoparticles deposition with PDDS and PSS and the deposition of magnetite and core-shall mesoporous silica nanoparticles. Finally different characterization of the modified sample is carried out like X-ray diffraction pattern using Bruker D8 Advance ECO powder diffract meter with $\mathrm{Cu}-\mathrm{Ka}$ radiation. Vibrating sample magnometer by quantum design and TEM image were obtained from tecnai T12 sprite microscope also be used. Scanning electron microscope using EDX system, thermo-gravimetric using TA instrument, and Fourier transform spectroscopy in Nicolet magna 760 FTIR spectrometer were used under this work.

\section{For functional finishing of cotton fabrics using zinc oxide-soluble starch nanocomposites}

reagents were of analytical grade without further purification are used with cotton fabrics and soluble starch to synthesize nano$\mathrm{ZnO}$ then characterize nano-ZnO using UV-visible spectrum in a Specord 50 ANALYTIKJENA® spectrophotometer, from 200 to 900 $\mathrm{nm}$, Photoluminescence spectra were recorded in a Perkin Elmer LS55® Spectrofluorimeter using 90 • illumination, the amount of soluble starch that was bound with the $\mathrm{ZnO}$ nanoparticles was obtained from a thermo-gravimetric, the x-ray diffraction (XRD) pattern of nano-ZnO was analyzed with a PANalytical X'pert PRO MPD $®$ X-ray diffractometer, The nano-ZnO samples were mounted on specimen stubs and examined with a Philips® XL 30 scanning electron microscope (SEM). Coating of cotton fabrics with nano$\mathrm{ZnO}$ then characterization of nano-ZnO coated cotton fabrics with elemental analysis like an atomic absorption spectrometer using an Avanta ${ }^{\circledR}$ PM unit, scanning electron microscopy, The antibacterial activity of cotton fabrics impregnated with nano-ZnO, evaluation was carried out with Staphylococcus aureus (ATCC 6538), a Grampositive bacterium and Klebsiella pneumoniae (ATCC 4352), a Gram-negative bacterium. The ability of a fabric to block UV light is given by the ultraviolet protection factor (UPF) values and UPF values are calculated according to AATCC test method.

\section{For durable anti-pilling modification of cotton fabric with chloropyrimidine compounds}

Plain woven and bleached cotton fabric and all the reagents used were analytically pure and used without any further purification. Stable and durable dispersed emulsions of chloropyrimidine compounds were prepared by the high shear emulsification method then preparation of ChloropyrimidineModified cotton fabric after making the cotton clean to make it free 
from any contamination. The control and modified cotton fabrics were dyed with reactive dyestuffs by an exhaustion method to observe the dyeing characteristics. Finally characterization antipilling behavior, surface morphology, FTIR spectra, XRD powder patterns, thermal stability, heat release property of modified and control cotton fabrics was evaluated by modified Martindale method ISO 12945-2: 2000, scanning electron microscope, FTIR instrument (Nicolet 5700, Thermo Fisher Scientific Inc., New York, NY, USA), Philips X'pert-pro MRD, Pyris Diamond TG-DTA thermal analyzer, FTT0001 Micro Calorimeter Combustion (MCC) instrument respectively. also washing durability, tensile properties, bending and surface friction properties test for the cotton samples was conducted according to the standard AATCC61-2006 method in the Wash Tec-P Fastness Tester, Instron 3365 Universal Testing Machine. Kawabata Evaluation System for Fabric (Kato Tech Co., Ltd., Kyoto,Japan) accordingly. The colorfastness of rubbing and washing with soap was determined according to ISO 105-X16 and ISO 105-C10.

All the chemicals used were of analytical grade with pretreated plain weaved gray cotton sample then irradiation is done by expose cotton to absorbed doses of 2, 4, 6, 8, and $10 \mathrm{kGy}$ using Cs137 gamma irradiator and mercerization was performed using different solutions of $\mathrm{NaOH}$. For comparative study with gamma ray treatment after bleaching, mercerized fabric was dyed at various temperatures and subjected to CIE lab system for the evaluation of color strength to get optimum concentration of alkali for mercerization. Dyeing of the cotton fabric (mercerized or gamma irradiated) was performed using the exhaust method and the dyed fabrics (gamma irradiated or mercerized) were analyzed using Spectraflash SF600, finally evaluation of characteristics of fabrics like the color fastness to washing, light, and rubbing of the dyed fabrics. The ISO methods IS0105BO2 (for light fastness), IS0105C03 (for washing fastness), and IS0105X-12 (for rubbing fastness), ASTMD 5034 (tensile strength test), and ASTMD 1424(tear strength), weight loss percentage in calculation of the dyed fabrics were used by Zahid M, et al. [10].

\section{Result and Discussion}

\section{For functionalized cotton fiber with 3-glycidoxypropyltriethoxysilane}

Functionalized Cotton Fiber with

3-Glycidoxypropyltriethoxysilane is due to monomer concentration on modification of cotton fiber results percent graft yield increased with an increase of silane concentration up to $400 \%$ for GPTES, with the increase of $\mathrm{pH}$ value up to 3.5 and ethanol/water ratio up to 40:60for GPTES and then decreased, with the increase of reaction temperature up to $30{ }^{\circ} \mathrm{C}$ for GPTES and then decreased with further increase of temperature. The dye exhaustion of the GPTES-modified cotton fiber was higher than that of unmodified washed cotton fiber and the dye exhaustion increased with an increase in the percent graft yield. The FTIR spectra of unmodified and silane-modified cotton fibers were mostly similar, except the new at $860 \mathrm{~cm}-1$ and $1207 \mathrm{~cm}-1$ for Si-OH symmetric stretch and Si-O-C bond, respectively. Surface roughness of the GPTES-modified cotton fiber is higher with a broad peaks and it became more amorphous as a consequence of further hydrolysis of the crystalline regions of cotton. The unmodified cotton fibers with silane coupling agents, there is a decrease of the swelling in the polar solvents and an increase in the non polar solvent. The thermal stability tensile strength, wrinkle recovery angle and flexibility of modified cotton fiber were higher than that of unmodified cotton fiber. Since the treated fiber sites are blocked the fiber changed to less affinity for moisture.

\section{For surface modification of cotton fabrics using plasma technology}

When the gray cotton fabric was treated in air plasma the weight loss is $1.12 \%$ for one minute of treatment, which is rather low but as the time of treatment was increased, the loss of weight started rising to $6 \%$ which is much higher than desized, scoured, and bleached samples. The morphology of treated surfaces look damaged or abraded due to etching. Crystalline index was increases from 0.3 to 0.6 and time of absorption decreased from 3600 sec for the gray fabric to less than a few seconds when the plasma treatment was carried out for about eight minutes. Using air plasma lead to formation of $\mathrm{C}=\mathrm{O}$ or $\mathrm{C}-\mathrm{N}$ bonds or the breaking of $\mathrm{CH}$ bonds, which increased wet ability and wick ability as well as the enhancement in the surface roughness properties. When reactive and natural dye was used the color strength increased after the plasma treatment, whereas for the direct dye there was a decrease in the strength because of etching away of the amorphous regions during the plasma treatment. The SEM photomicrographs showed fibril-like formations on the surface and the contact angle decreased from $139^{\circ}$ to that corresponding to the control fabric $122^{\circ}$ after five washes and there is a loss in the tensile strength from 52 to $40 \mathrm{MPa}$ after treatment for eight minutes. According to X-ray photoelectron spectroscopic there is incorporation of oxygen-containing moieties on the surface, as evidenced by the enhanced intensity of the peak at $285.6 \mathrm{eV}$.Fourier transform infrared-attenuated total reflectance studies shows the spectra that the absorption bands due to $\mathrm{O}-\mathrm{H}$ and $\mathrm{C}-\mathrm{H}$ bonds are clearly seen at 3270 and 2925 per $\mathrm{cm}$ at these peaks decrease considerably on treatment with DCFM plasma. Similarly, the areas of absorption bands appearing at 1743 and 1422 per $\mathrm{cm}$ also decrease after the DCFM treatment. Thus it appears that $\mathrm{O}-\mathrm{H}$, $\mathrm{C}-\mathrm{H}$ bonds in the cellulose are getting scissoned and $\mathrm{H}$ is being replaced with either $\mathrm{F}$ or $\mathrm{Cl}$ from the DCFM.

\section{For super hydrophobic cotton textile with robust composite film and flame retardancy}

Amine zinc oxide and $\mathrm{ZnO}$-coated films is formed after fluorinations of the cotton surface. During covalent deposition, numerous embossment caused by $\mathrm{ZnO}$ at the submicron level had been emerging and creating a rough film on the fiber surface 
and the $\mathrm{ZnO}$-coated textile transformed from super hydrophilic to super hydrophobic with a WCA of $158 \pm 1^{\circ}$ after fluorination, show no obvious change on the size and morphology of the ZnO attached to the sample surface. The combination of roughness with $\mathrm{ZnO}$ and layer with lower surface energy is pivotal for achieving a super hydrophobic cotton fiber with polystyrene to decrease roughness. Since flammability of cotton in $18.3 \%$ is limited to industrial applications so the epoxy resin and APDMS treatment increase to $21 \%$. Zinc oxide, coupling agent nano composite film show the most effective for flame retrardance and thermal stability than epoxy resin per fluorocarbon silane and polystyrene. The thermal degradation after $5 \mathrm{~min}$ ultrasonic washing of cotton fiber treated with ZnO film without PS or epoxy resin is lower. The abrasion resistance test has been slightly damaged with a WCA of $145^{\circ}$, showing the outstanding abrasion resistance and supper hydrophobic durability. A chemical stability of the sample didn't show visible change although both sample before and after PS treatment display excellent separation process.

\section{For fabrication of super hydrophobic cotton fabrics by a simple chemical modification}

Samples of fabrics were modified in a one-step process or a two-step process. The modification were carried out at room temperature, $30 \mathrm{~min}$ and at $80^{\circ} \mathrm{C}$. The differences in add-on values between all samples studied are not large. The contact angle measurements show that all the modified samples are strongly hydrophobic. However, after the washing process the mentioned WCA decreased, contrary to the WCA on our sample that increased after washing. Moreover, a higher temperature of the process favors a more efficient hydrolysis and condensation on the fiber surface as results from the fact that in all cases higher values of the contact angle were observed for the modification proceeding a $80^{\circ} \mathrm{C}$, when it was carried out as both the one-step or the two-step process and the contact angle values increased after washing. Silicon and fluorine contents determined after multiple washing are close to those before washing, which testifies to a durable attachment of modifiers to the fiber surfaces. Fabrics impregnated with the composition containing polysiloxane 4 contain more fluorine than those modified with polysiloxane 1 , which results from the higher fluorine content in polysiloxane 4 used for the impregnation. However, despite the higher fluorine content, WCA on this fabric appeared to be smaller, as was already mentioned.SiO2 clusters were incorporated in the structure of fiber, making their surface heterogeneous that is why polysiloxane 1 has better properties (flexibility, bending)soft hand and had not changed their color, and no loss of the modifiers layer and hardening than polysiloxane 2 , because of more fluoroalkyl groups and fewer trialkoxysilyl ones therefore, one-step modification gives strongly hydrophobic properties. These properties are durable and are maintained after ten washings. None of the one-step modification affected the appearance and structure of fabrics.

\section{For modification of cotton fiber with functionalized silane coupling agents vinyltriethoxysilane and amino propyltriethoxysilane}

The silane coupling agents on modified cotton fiber increase weight gain with the increase of silane concentration for VTES and APTES and decreased with a further increase in the monomer concentration was due to the higher cross-linking reaction between the $-\mathrm{OH}$ groups of cellulose and $-\mathrm{OH}$ groups of the silane coupling agents at higher concentration and increasing rate of diffusion of monomer into the fiber structure also leading to a higher graft yield. Slowly increasing the silane concentration definitely increases the number of reactive groups in the reaction medium the maximum graft yield was obtained when the cotton fiber was treated with $500 \%$ vinyltriethoxysilane and $600 \%$ aminopropyltriethoxysilane. Weight gain percentage increases with the increase of $\mathrm{pH}$ value and then starts to decrease and the hydrolysis process was facilitated by homogenizing agents. At an ethanol to water ratio of 40:60 for VTES and 80:20 for APTES, the weight gain after modification reached the optimum value. Weight gain increased with the increase of time, up to 90 minutes, both for VTES and APTES. The FTIR spectra of raw and silane-modified cotton fibers are mostly similar. Modified fiber has as smoother surface than that of raw fiber, due to the homogeneous deposition of the silane layer on the fiber backbone. After modification, the existence of Si-O-Si and $\mathrm{Si}-\mathrm{O}-\mathrm{C}$ bonds on the fiber surface was identified by the FTIR analysis. The VTESand APTES functionalized cotton fiber contains1.9 and 4.2 weight percent of silicon. After treating with silane coupling agents, there is a decrease of the swelling in the polar solvents but an increase in the nonpolar solvent. The tensile strength of modified cotton fiber was higher than that of raw cotton fiber. The moisture absorption sites are blocked after incorporation of silane, through surface modification, reducing the affinity for moisture and the dye exhaustion of the silane-modified cotton fiber was higher than that of the raw cotton fiber and the dye exhaustion increased with an increase in the percent graft yield.

\section{For modification of cotton fibers with sericin using non- formaldehyde released cross linking agents}

The percentage of add-on of samples finished with GD and DMeDHEU sharply increases with increasing concentration of sericin up to $100 \mathrm{~g} / \mathrm{L}$ sericin; the increased tendency of percentage of add-on shows that sericin can be used for the modification at higher concentration. FTIR-ATR spectrum of samples treated with GD only is identical to that of the untreated ones while that of the samples treated with the combination of GD and sericin is distinctly different. Cotton fibers cylindrical when treated with glutaraldehyde and DMeHEU. SEM images show that sericin molecules are bound on cotton fiber surfaces. The color strength of samples substantially increased for samples treated with $25 \mathrm{~g} / \mathrm{L}$ sericin, especially when DMeDHEU was used since react with amino group in sericin backbone, and hydroxyl and carboxylic groups in the side group of 
sericin through methylol group while aldehyde group of GD reacts with only hydroxyl group of sericin and continuously increase with increasing sericin concentration up to $75 \mathrm{~g} / \mathrm{L}$ up to $100 \mathrm{~g} / \mathrm{L}$ sericin.

\section{For modification of cotton fibers with magnetite and magnetic core-shell mesoporous silica nanoparticles}

Characterization of the nano particles to deposition of the nano particles on the cotton sample in aqueous solution and the attraction is predominated vander walls force, easy remotion of nano particle from surface cause shifting to electrostatic attraction force. According to XRD pattern of sample cotton fibers with magnetite and magnetic core-shell mesoporous silica nanoparticles indicate a weekend intensity of the representative peaks and the overlapping of cotton functional groups. The thermal analysis show small weight residues remain at $600{ }^{\circ} \mathrm{C}(7.76$ and $5.53 \%$ of the initial weight respectively) the remaining weight percentages can be attributed to the presence of inorganic material.

\section{For functional finishing of cotton fabrics using zinc oxide-soluble starch nanocomposites}

The high repulsive forces between the lone pair of electrons of two oxygen atoms of zinc hydroxide in the formation of $\mathrm{ZnO}$ nanoparticles. The simultaneous presence of soluble starch in the reaction medium quickly protects the formed $\mathrm{ZnO}$ from further agglomeration, resulting in the formation of $\mathrm{ZnO}$-soluble starch nanocomposites. Thermo-gravimetric analysis of $\mathrm{ZnO}$ nanoparticles synthesized with $0.5 \%$ soluble starch showed a weight loss of $27.64 \%$ at around $312{ }^{\circ} \mathrm{C}$, which matches with the degradation temperature of starch and width of the peaks in the case of nano-ZnO has increased due to the quantum size effect.TEM measurements, indicating a strong binding between the $\mathrm{ZnO}$ and soluble starch the FTIR spectra recorded the peaks corresponding to soluble starch, indicating the strong binding of soluble starch with the $\mathrm{ZnO}$ nanoparticles. But no covalent bond formed between $\mathrm{ZnO}$ and soluble starch as analyzed by FTIR spectra. Nano-ZnO coating with $1.63 \%$ uptake of $\mathrm{ZnO}$ showed excellent antibacterial activity (reduction > 99.9\%), even better than bulk $\mathrm{ZnO}$ because of presence of soluble starch. $\mathrm{ZnO}$ has been found to have several advantages, including marked antibacterial activity in the neutral region $(\mathrm{pH}=$ 7) without the presence of light, and being non-toxic to humans. The nano-ZnO coated cotton fabrics showed efficient blocking of UV radiation in both regions. The nano-ZnO impregnated fabrics were found to retain more than $80 \%$ efficiency of both antibacterial and UV-protection functions even after 25 wash cycles.

\section{For modification of cotton fabric with chloropyrimidine compounds}

Dong $X$, et al. [9] done durable anti-pilling modification of cotton fabric with chloropyrimidine compounds were carried out anti-pilling grade of the modified cotton fabric increased gradually with the concentration of TLP dispersions ranging from $0.5 \mathrm{~g} / \mathrm{L}$ to $3.0 \mathrm{~g} / \mathrm{L}$. However, when it reached $4.0 \mathrm{~g} / \mathrm{L}$, the anti-pilling grade remained the same and even decreased a little. Na2CO3 is the optimum factor, and stronger alkali conditions were not conducive to the covalent cross linking of cotton fabric with chloropyrimidine compounds. The anti-pilling grade of the modified cotton fabric did not change markedly when the concentration of $\mathrm{Na} 2 \mathrm{CO} 3$ and Na2SO 4 varied from 2 to $4 \mathrm{~g} / \mathrm{L}$ so; $3 \mathrm{~g} / \mathrm{L}$ was the most suitable process parameter at temperature of $95^{\circ} \mathrm{C}$ and the time of $45 \mathrm{~min}$, because longer time or higher temperature can seriously reduce the mechanical properties of cotton fabric. Even if dense layer of film is formed on the surface of the modified fiber the cotton fabric surface became rough and a fabrics held together more tightly and did not easily fall of and separate, because of the cross linking between fiber and chloropyrimidine compounds. Control cotton fibers present typical convoluted and wrinkle-like structure due to the longitudinal fibril structure. The cellulose chains of cotton did not change after modification by chloropyrimidine compounds. However, a new absorption band of modified cotton fabrics visibly appeared at 1580-1520 cm-1 assigned to the stretching vibration of $\mathrm{C}=\mathrm{C}$ and $\mathrm{C}=\mathrm{N}$ double bonds of the six-membered ring on chloropyrimidine compounds and the peak at $3347 \mathrm{~cm}-1$ for $\mathrm{O}-\mathrm{H}$ deformation stretching decreased slightly after the modification with chloropyrimidine compounds, which further confirmed that the nucleophilic substitution reaction existed between cotton fabric and chloropyrimidine compounds. According to X-ray diffraction compounds modified cotton samples retained the original crystalline form of pristine cotton fabric regardless of the heterogeneous modification. The anti-pilling grade of the control cotton fabric is 2-3, while the TLP and ADP-modified cotton fabrics are classified as grade 5 , indicating that there is almost no pilling on the surface of the modified cotton fabrics under 2000 pilling cycles. Fabrics treated with chloropyrimidine compound emulsions, the pilling grade increased to 5 or 4-5 before washing, indicating the good emulsion stability and presence of chloropyrimidine compounds as an effective cross linking agent.DTG of a control cotton fabric had the fastest decomposition rate at $349^{\circ} \mathrm{C}$ and TLP-, DMP-, and ADPmodified cotton had maximum degradation at $339^{\circ} \mathrm{C}, 343{ }^{\circ} \mathrm{C}$, and $342{ }^{\circ} \mathrm{C}$, respectively. The tensile strength and elongation at break of modified cotton fabrics in warp direction decreased slightly and air permeability of the modified cotton fabrics also had a slight decrease due to the chloropyrimidine film formed on the surface of fiber. It was interesting that the whiteness of TLPCF and DMPCF improved but the whiteness of ADPCF decreased sharply because the yellow color of the ADP reagent itself affected the whiteness. The bending properties of TLPCF and DMPCF had no obvious change and maintained a soft and smooth handle. Since modified cotton fabrics dyed with Reactive Red3BF showed similar $L^{*}, a^{*}, b^{*}, C^{*}$, and Hvalues, which mean the modification had little effects on the dyeing of cotton fabrics. Washing fastness (fastness and staining) of dyed TLPCF, DMPCF, and ADPCF was at the same level as that of control cotton fabric. The graft modification of chloropyrimidine compounds did not affected the dyeing fastness of cotton fabrics and even improved the wet rubbing fastness. 


\section{For modification of cotton fabric for textile dyeing: industrial mercerization versus gamma irradiation}

The fabric was irradiated for different absorbed doses to screen the best gamma-ray dose for coloring the textile at various temperatures $\left(50,60,70,80\right.$, and $\left.90{ }^{\circ} \mathrm{C}\right)$ in order to optimize the temperature. The influence of the dyeing temperature on the color strength of irradiated fabric to the absorbed dose of 2-10 kGy is shown to obtain maximum color yield. The color strength initially increased with the rise in temperature up to $70^{\circ} \mathrm{C}$ and then decreased as the dyeing was carried out above $70{ }^{\circ} \mathrm{C}$. Therefore, $70{ }^{\circ} \mathrm{C}$ was the optimum temperature for the dyeing of irradiated cotton fabric ( $2 \mathrm{kGy}$ ). After caustisization of fabric using various amount of at optimum alkali $20 \%$, followed by dyeing at different temperature Low amount of alkali did not remove the greasy substance so it gives low color depth. While high amount of alkali used loosening of fabric strength, which also results in failure of dye uptake. Gamma irradiated cotton fabric requires less severe conditions for dyeing and gives better color strength values due to space between cellulose fiber is increased on radiation treatment this result the color strength decreased and uneven shade appeared due to desorption of dye and attachment of impurities along with dye molecules and the color fastness properties were improved from fair (mercerized fabric 20\%) to good (irradiated cotton $2 \mathrm{kGy}$ ) under optimum conditions of $\mathrm{pH}$, temperature, salt concentration, and dyeing time. The irradiation might cause degradation of the cotton cellulosic material to a small extent, and thus slightly decreasing the mechanical strength of the fabric as compared to conventional mercerization.

\section{Application}

- Modification of Cotton Fabrics Using Plasma Technology is necessary for different application were hydrophobic surface needed.

- Modification of cotton fibers with magnetite and magnetic core-shell mesoporous silica nanoparticles is needed to use were high affinity of dye necessary in industrial or other functional application.

- It is useful to modify cotton fibers with sericin, and use them for medical textiles applications.

- Textile Performance of Functionalized Cotton Fiber with 3-Glycidoxypropyltriethoxysilane maximum to improve physicochemical properties such as tensile properties, moisture absorption, which suggest the possibility to apply this method in the production of garment products, textiles etc.

- Functional finishing of cotton fabrics using zinc oxide-soluble starch nanocomposites used to use cotton for applications such as UV-protection ability in textiles and sunscreens, and antibacterial finishes in medical textiles and inner wears.
- Modification of cotton fabric with chloropyrimidine compounds improving the antipilling behavior of cellulosic materials and supports further preparation of functional textiles.

- Modification of cotton fabric for textile dyeing: industrial mercerization versus gamma irradiation used where color strength needed like decoration since the coloring properties using high-energy gamma radiations for natural as well as synthetic dyes give excellent coloring property.

- $\quad$ Super hydrophobic cotton textile with robust composite film and flame retardancy used on industrial or technical application.

- Modification of cotton fiber with functionalized silane coupling agent's vinyltriethoxysilane and aminopropyltriethoxysilane for the production of hydrophobic cotton material for technical, medical and industrial applications.

\section{Conclusion}

With the improvement of living standards, people have higher and more complete requirements of textile quality and functionality to achieve such application different researcher report means of cotton material improvement the one were Mondal IH, et al. [1] in which they done chemical modification of cotton fibers with silane coupling agents' with optimum value of the reaction parameters, such as silane concentration, $\mathrm{pH}$, ethanol- water ratio and temperature shows an improvement in physicochemical properties such as tensile properties, moisture absorption. The formation of highly hydrophobic surface, due to thin polymeric fibril formations, by using plasma generation with DCFM gas treatment also reported by Bhat NV, et al. [2]. Cotton were also be modified with Przybylak M. et al. [4] to got super hydrophobic cotton fabrics by a simple chemical modification with bifunctional polysiloxanes containing fluoroalkyl groups (those imparting surface properties) and trialkoxysilyl or glycidyl groups (those bonding to substrates) were show good hydrophobizing agents. A very simple one-step method of fabric modification was developed (by impregnation in solutions of the aforementioned polysiloxanes) makes it possible to produce super-hydrophobic fabrics, properties of which are in durable, cheap, simple and fast (30 min impregnation) way. Ming zhang, et al., 2015, also made super hydrophobic modification cotton textile with covalent deposition to prepare a robust zinc oxide film on the cotton surface with a WCA of $158 \pm 1^{\circ}$, and its LOI value greatly increased from $18.3 \%$ to $21.6 \%$, showing its outstanding hydrophobicity, flame retardancy and thermal stability and super hydrophobic cotton fabric, weather mechanically or chemically further stabilize through addition of polystyrene. However, according to Mondal IH, et al. [1] improve physicochemical properties such as tensile properties, moisture absorption using textile performance of functionalized 
cotton fiber with 3-Glycidoxypropyltriethoxysilane maximum weight gain percent is obtained at the optimum value of silane concentration, $\mathrm{pH}$, ethanol-water ratio and time. When a Cotton Fibers modified with Sericin Using Non-Formaldehyde Released Cross linking Agents by Kongdee A, et al. [6] the affinity of dye for samples increased with increasing sericin due to the decreased in $\mathrm{L}^{*}$-values and increased in $\mathrm{K} / \mathrm{S}$ and $\mathrm{b}^{*}$ values and the samples treated with DMeDHEU yielded greater K/S. Patino-Ruiz D, et al. [7] also reports a result on modification of cotton fibers with magnetite and magnetic core-shell mesoporous silica nanoparticles through SEM and TEM image, XDR and EDS patterns and nanoparticles layers on the cotton surface verify that the cotton modified with Fe304 nanoparticles had a good magnetization response, whereas the cotton modified with $\mathrm{Fe} 304-\mathrm{mSiO} 2$ nanoparticles showed a reduced magnetization response.

Functional finishing of cotton fabrics using zinc oxide-soluble starch Nano-composites with Vigneshwaran $\mathrm{N}$, et al. [8] were reported UV-protection and antibacterial property is improved because of $\mathrm{ZnO}$ nanoparticles embedded in polymer matrices and when cotton fabrics were modified with chloropyrimidine compounds by Dong X, et al. [9] anti-pilling performance is improved by two or more grades before and after washing with optimized factors and modified cotton fabrics exhibited good handle, whiteness, heat release behavior, and dyeing properties without significant damage to the physical and mechanical properties Other modification like gamma-ray treatment of cotton fabric for textile dyeing with Zahid M, et al. [10] were showing an improvement of the color strength and the shade of Reactive Violet H3RF and has potential to be studied at the industrial scale. Gamma irradiation has also shown comparable color fastness properties (to that of mercerization) of the dyed cotton fabrics.

\section{Acknowledgement}

None.

\section{Conflict of Interest}

Author declare no conflict of interest.

\section{References}

1. Mondal IH, Islam K, Ahmed F (2018) Textile Performance of Functionalized Cotton Fiber with 3-Glycidoxypropyltriethoxysilane. J Textile Sci Eng 8(1): 337.

2. Bhat NV, Netravali AN, Gore AV, Sathianarayanan MP, Arolkar GA, et al. (2014) Surface Modification of Cotton Fabrics Using Plasma Technology. Textile Research Journal 81(10): 1014-1026.

3. Zhang M, Zhang D, Shi J, Gao Z, Wnag C, et al. (2015) Super hydrophobic cotton textile with robust composite film and flame retardancy. RSC Advances 5: 67780-67786.

4. Przybylak M. Maciejewski H, Dutkiewicz A, Dąbek I, Nowicki M (2016) Fabrication of super hydrophobic cotton fabrics by a simple chemical modification. Cellulose 23: 2185-2197.

5. Mondal MIH, Islam MK, Ahmed F (2018) Modification of Cotton Fibre with Functionalized Silane Coupling Agents Vinyltriethoxysilane and Aminopropyltriethoxysilane. J Textile Sci Eng 8(3): 1-8.

6. Kongdee A, Chinthawan N (2007) Modification of Cotton Fibers with Sericin Using Non-Formaldehyde Released Cross linking Agents. RJTA 11(3): 18-26.

7. Patino-Ruiz D, Sanchez-Botero L, Hinestroza J, Herrera A (2018) Modification of cotton fibers with magnetite and magnetic core-shell mesoporous silica nanoparticles. Journal of Phys Status Solidi A 215(19).

8. Vigneshwaran N, Kumar S, Kathe AA, Varadarajan PV, Prasad V (2006) Functional finishing of cotton fabrics using zinc oxide-soluble starch nanocomposites. Journal of Nanotechnology 17: 5087-5095.

9. Dong X, Xing T, Chen G (2019) Durable Antipilling Modification of Cotton Fabric with Chloropyrimidine Compounds. Polymers 11(10): 1697.

10. Zahid M, Bhatti IJ, Adeel S, Saba S (2016) Modification of cotton fabric for textile dyeing: industrial mercerization versus gamma irradiation, The Journal of The Textile Institute 108(2). 